POS $\quad$ PROCEEDINGS

\title{
Constraints to the GRB central engine from jet penetrability to massive stars
}

\author{
Hiroki Nagakura ${ }^{a, b *}$, Yudai Suwa ${ }^{a}$ and Kunihito loka ${ }^{c, d}$ \\ ${ }^{a}$ Yukawa Institute for Theoretical Physics, Kyoto University, Kyoto 606-8502, Japan \\ ${ }^{b}$ Department of Science and Engineering, Waseda University, Tokyo 169-8555, Japan \\ ${ }^{c}$ KEK Theory Center, 1-1 Oho, Tsukuba 305-0801, Japan \\ ${ }^{d}$ Department of Particle and Nuclear Physics, The Graduate University for Advanced Studies \\ (Sokendai), 1-1 Oho, Tsukuba 305-0801, Japan \\ E-mail: hiroki@heap.phys.waseda.ac.jp
}

\begin{abstract}
We investigate the neutrino-driven jet penetrability by performing two dimensional relativistic hydrodynamic simulations. The jet luminosity is determined by the neutrino energy deposition rate around a black hole. We take into account the evolution of mass accretion rate, black hole mass and spin, all of which are necessary to determine the luminosity of jet. We show that the neutrino-driven jet succeeds to break out the current elaborate Wolf-Rayet progenitor. After the jet breakout, we further investigate the evolution of jet with the analytical treatment and it turns out that the jet potentially produces emissions with isotropic-luminosity $L_{p(i s o)} \sim 10^{52} \mathrm{erg} / \mathrm{s}$ and isotropic-energy $E_{j(\text { iso })} \sim 10^{54} \mathrm{erg}$, which are sufficiently large to explain GRBs. We also investigate the dependence of the rate of progenitor rotation, and reveal that the neutrino-driven jet is capable to generate the various types of explosion such as GRBs, low luminosity GRBs or $\mathrm{X}$-ray Flashes and failed GRBs.
\end{abstract}

Gamma-Ray Bursts 2012 Conference-GRB2012,

May 07-11, 2012

Munich, Germany

\footnotetext{
*Speaker.
} 


\section{Introduction}

The neutrino-driven collapsar model is one of the most plausible scenarios for the origin of GRBs [1]. Over the years, substantial works have been made to understand if the neutrino-driven collapsar can produce GRBs. However, there are still several uncertainties to obtain reliable statements. One of the largest uncertainties is the efficiency of energy deposition by neutrinos. A number of studies have conducted to estimate the efficiecy by employing the steady state approximation (see e.g. [2, 3]). Recently, Zalamea \& Beloborodov [3] have conducted General Relativistic Ray Tracing Neutrino Radiation Transfer and they found that the deposition energy by neutrinos could be well described as a simple analytic formula. Thanks to these studies, the jet luminosity can be estimated qualitatively without expensive numerical simulations.

It should be noted, however, even if the neutrino-driven jet operates, it does not correspond to the success of GRB. As the minimum requirement, the jet should penetrate the stellar envelope. Otherwise the jet becomes non-relativistic ejecta and incapable of producing GRBs. Ever since the neutrino-driven collapsar model was proposed, a number of numerical studies have been done (see e.g. [4, 5]). Among these studies, however, the jet luminosity is assumed to be constant or the constant conversion efficiency from the accretion energy [4]. In order to judge whether or not the neutrino-driven jet is capable of penetrating through the progenitor, we need to investigate the jet penetrability powered by neutrino energy deposition.

Here we present the first results studying neutrino-driven jet propagation calculating the evolution of the mass accretion rate and black hole mass and spin, all of which are necessary to evaluate the energy deposition by neutrinos. The purpose of this study is to clarify whether the nuetrinodriven jet can succesfully propagate through and break out of the stellar surface. In addition, we also investigate how the angular momentum distribution of the progenitor star affects the final fate of the explosion. As shown below, the final outcome of the explosion depends on the rate of rotation and differences in the angular momentum of stars may be the origin of the observational differences such as GRBs, Low luminosity GRBs or X-ray Flashes and failed GRBs.

\section{Methods and Models}

We perform two dimensional axisymmetric (and also equatorial symmetric) simulations of relativistic hydrodynamics for the envelope accretion and jet propagation. The numerical code employed in this paper is essentially the same as those used in previous papers $[4,5]$. The initial stellar density distribution is fixed as 16TI model in [6]. The mass accretion rate $(\dot{M})$ is estimated by the mass flows through the inner boundary (see Eq. (1) in [4]). The mass and angular momentum in the excised region are assumed to be the same mass and angular momentum of the black hole. The time evolution of mass and spin of black hole is calculated by integrating mass and angular momentum flux crossing the inner boundary. It should be noted that when the specific angular momentum (SAM) at the location of inner boundary in equatorial region becomes larger than SAM at the inner most stable circular orbit (ISCO) (see cross marks in Figure 1), we change the rule to integrate the angular momentum as; $f_{a}=\dot{M} \times J_{I S C O}$, where $f_{a}$ and $J_{I S C O}$ denote the angular momentum flux and SAM of ISCO, respectively. The jet luminosity is determined by the analytic 


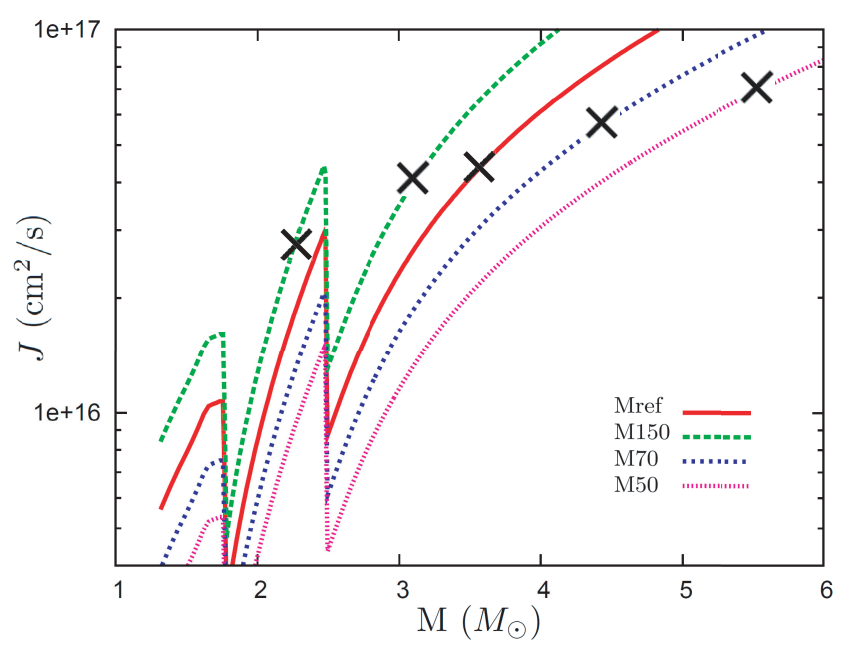

Figure 1: SAM distribution of models (Red: Mref, Green: M150, Blue: M70, Pink: M50). The cross marks denote the intersection between the SAM of star and one for the last stable orbit for a black hole with the mass and angular momentum inside the indicated coordinate.

formula obtained by [3]. We assume that the jet is injected only when the SAM of matter at the inner boundary in equatorial plane exceeds $J_{I S C O}$.

We employ the gamma-law equation of state with $\gamma=4 / 3$. The spherical symmetric density distribution is mapped by the spherical coordinate. The computational domain covers from $10^{8}$ to $4 \times 10^{10} \mathrm{~cm}$. The jet injection parameter such as Lorentz factor and the specific internal energy is the same as those used in the standard model of [4]. We set up the rotation by the similar manner as those used in [7]. The SAM distribution is separated into radial and polar components as $J(r, \theta)=j(r) \Theta(\theta)$, where $r$ and $\theta$ are the spherical radius and polar angle, respectively. As reference model (Mref), $j(r)$ is given by 16TI model. For M150, M70 and M50 models, $j(r)$ is multiplyed one for the reference model by 1.5, 0.7 and 0.5, respectively (see Figure 1). The polar angle components are assumed to be rigid body rotation on shells, i.e., $\Theta(\theta)=\sin ^{2} \theta$.

\section{Results}

Table 1 shows the summary of our results. The foremost important result in this study is that the jet succeeds to break out from the star except for model M50. For model M50, the forward shock wave does not move out and almost stagnates around the inner boundary despite the successful operation of the central engine (Note that $t_{i}$ in Table 1 denotes the time of initiation of central engine). This is attributed to the fact that the jet power does not exceed the ram pressure of accretion flows. For models with successful jet breakout, the jet also can not move forward soon after the initiation of the central engine. However, by a rise in spin parameter of the black hole over time, the jet power eventually exceeds the ram pressure of accretion flows and succeeds to penetrate the star.

Figure 2 shows the evolution of hemispherical neutrino luminosity, mass accretion rate, black hole mass, spin parameter and conversion efficiency from accretion energy to neutrino luminosity 
Table 1: Summary of our results

\begin{tabular}{lcccccccc}
\hline \hline Model & Breakout & $\begin{array}{c}t_{i} \\
(\mathrm{~s})\end{array}$ & $\begin{array}{c}t_{b r} \\
(\mathrm{~s})\end{array}$ & $\begin{array}{c}L_{p} \\
\left(10^{50} \mathrm{erg} / \mathrm{s}\right)\end{array}$ & $\begin{array}{c}E_{d g} \\
\left(10^{51} \mathrm{erg}\right)\end{array}$ & $\begin{array}{c}E_{j} \\
\left(10^{51} \mathrm{erg}\right)\end{array}$ & $\begin{array}{c}E_{j>L_{50}} \\
\left(10^{51} \mathrm{erg}\right)\end{array}$ & $\begin{array}{c}E_{j>L_{49.5}} \\
\left(10^{51} \mathrm{erg}\right)\end{array}$ \\
\hline Mref & yes & 10.9 & 27.8 & 1.9 & 1.4 & 7.4 & 4.4 & 5.6 \\
M150 & yes & 2.2 & 17.6 & 3.2 & 1.6 & 11.7 & 8.8 & 9.8 \\
M70 & yes & 15.5 & 45.5 & 1.0 & 0.9 & 3.6 & - & 1.7 \\
M50 & no & 21.6 & - & 0.6 & - & - & - & - \\
\hline \hline
\end{tabular}

( $\eta \equiv L_{j} / \dot{M} c^{2}$ ) as a function of time from the onset of the collapse. It should be noted that, in order to see the evolution of these quantities after the jet break out, we employee the following analytic approximations (see e.g. $[4,8]) ; t=t_{(b)}+\int_{r_{(b)}}^{r} \frac{d t_{f f}}{d r}(r) d r$ and $M_{b h}=M_{b h(b)}+\int_{r_{(b)}}^{r} \frac{d M_{r}}{d r}(r) d r$, where $t_{f f}(r)=\beta \sqrt{\frac{G M_{r}}{r^{3}}}$ and $\dot{M}_{f f}(r)=\frac{d M_{r} / d r}{d t_{f f} / d r}=\frac{1}{\beta^{2}} \frac{8 \pi G M_{r} t_{f f} \rho}{3 M_{r}-4 \pi r^{3} \rho}$. Here, $t_{b}$ denotes the time at the jet break out. Note also that the functions of $M_{r}(r)$ and $\rho(r)$ are extracted from the table of 16TI model. $r_{b}$ is determined by the assumption of $M_{b h}\left(t_{b}\right)=M_{r}\left(r_{(b)}\right)$. The non-dimensional parameter $\beta$ is determined so as for $\dot{M}\left(t_{b}\right)$ to be equal to $\dot{M}_{f f}\left(r_{(b)}\right)$.

As shown in Figure 2, the neutrino luminosity and its peak values are higher for the faster rotation model. This is due to the fact that the kerr parameter becomes large and the central engine starts to operate in the early timing. The dependence of the neutrino luminosity on the kerr parameter is very huge, which becomes the chief cause of the different outcome of explosion. In addition, $M_{b h}$ does not grow and $\dot{M}$ is still high, all of which works to strengthen the power of jet. We also find that the time-averaged accretion-to-jet conversion efficiency among successful jet breakout models is roughly $\eta \sim 10^{-3}$, while $\eta$ for M50 can not reach $10^{-3}$ and never produces a jet breakout. This result is consistent with the previous work [4]. Therefore, we conclude that the criteria for jet penetrability in [4] can be applied to the neutrino-driven jet.

In order to discuss the outcome of explosion, the energetics of the jet is divided into two components: relativistic jet component $\left(E_{j}\right)$ and cocoon component, i.e. non-relativistic ejecta $\left(E_{d g}\right.$ ) (see [8]). After the jet breakout, the injected energy is assumed to go to the relativistic component, i.e, $E_{j}$ is given by $\int_{t_{b}}^{\infty}\left(L_{j} / 2\right) d t$ (we consider the hemispheric region for the relativistic jet component). On the other hand, the energy of cocoon component can be estimated as the diagnostic energy at $t=t_{b}$ (see [4]). It is important to note that, as shown in Table $1, E_{d g}$ is typically $\sim 10^{51} \mathrm{erg}$, which is an order of magnitude lower than the typical explosion energy of Hypernova. It may imply that the extra mechanism such as the disk wind by viscous-heating or magnetic-driven wind would be required to explain the link between the GRBs-Hypernovae.

As shown in Table $1, E_{j}$ increases with an increase of stellar rotation. For the purpose of studying the outcome of explosion in more detail, we further divide the energy of relativistic jet into $E_{j>L_{50}}$ and $E_{j>L_{49.5}} . E_{j>L_{50}}$ is calculated by the same manner as $E_{j}$ except for the integration is carried out when $L_{j} / 2>10^{50} \mathrm{erg} / \mathrm{s}$, while $E_{j>L_{49.5}}$ is calculated with a condition $L_{j} / 2>5 \times$ $10^{49} \mathrm{erg} / \mathrm{s}$. It is interesting to note that model M70, which is the slowest model among successful jet breakout models, does not have $E_{j>L_{50}}$ due to low luminosity jet. In addition, $E_{j}$ becomes quite lower than other models. According to this result, neutrino-driven jet from the compact Wolf-Rayet star with a rotation similar as model M70 would be favor progenitor for low luminosity GRBs or 

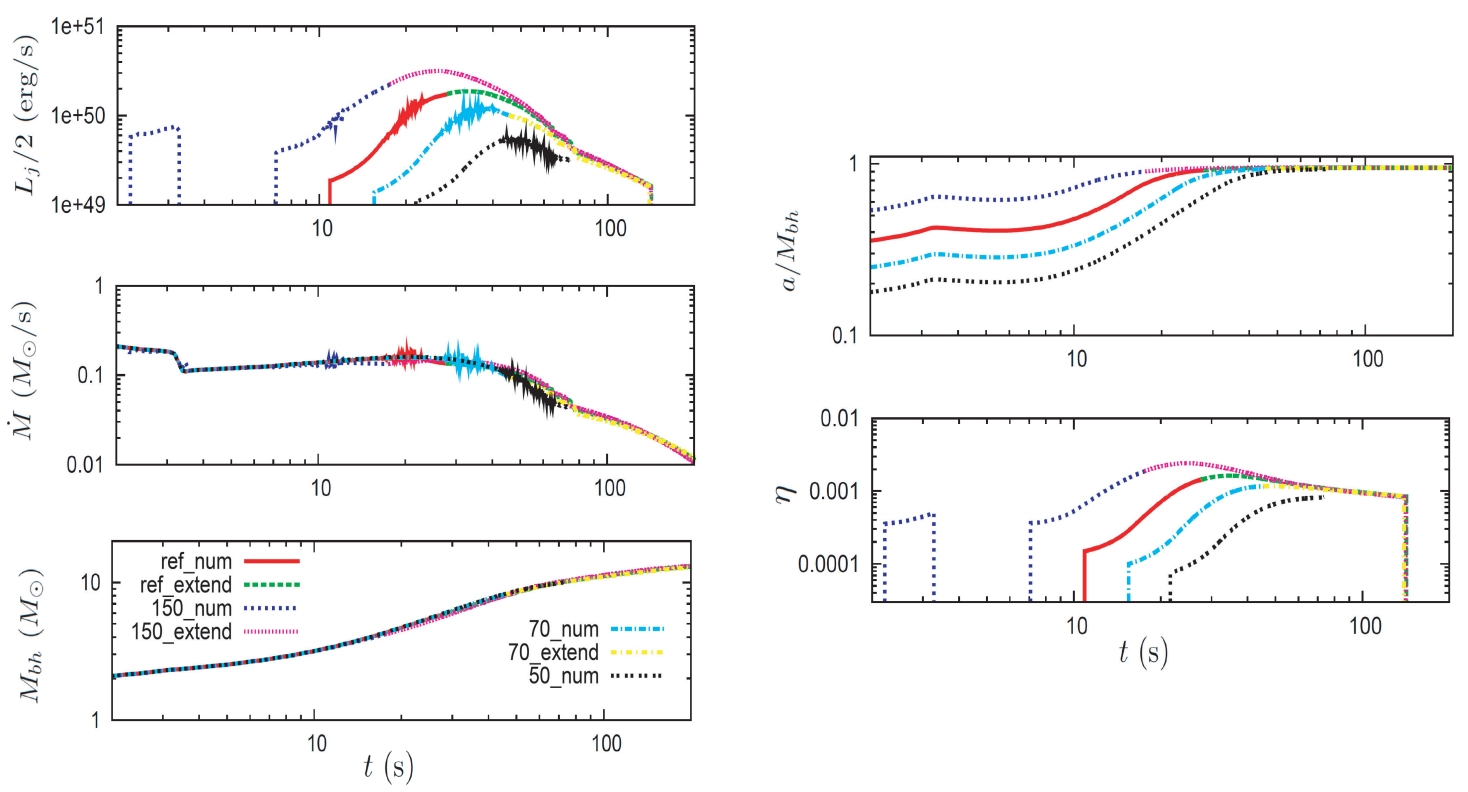

Figure 2: Hemispherical neutrino luminosity, mass accretion rate, black hole mass, spin parameter and conversion efficiency from accretion energy to neutrino luminosity $\left(\eta \equiv L_{j} / \dot{M} c^{2}\right)$ as a function of time from the onset of the collapse. Each color represents different models. Note that the model name with "extend" indicates the extrapolate results by analytic treatment (see text for more details).

might be X-ray flashes. It is also interesting to note that $E_{j}$ of model M70 is still $10^{51} \mathrm{erg}$, which is not so different from other models. This is due to the fact that the jet with slowly rotating core tends to be weak power and it spends long time to penetrate the star, eventually large fraction of jet energy has been consumed for sweeping aside the stellar mantle.

In order to compare observations, we calculate the isotropic energy $\left(E_{j(i s o)}\right)$ for $E_{j}, E_{j>L_{49.5}}$ and $E_{j>L_{50}}$, and also isotropic peak luminosity $L_{p(i s o)}$, which are shown in Figure 3. In this calculation, the jet opening angle is assumed to be $\theta_{o p}=9^{\circ}$, which is the same as the root of injected jet. Note that we neglect the effect of recollimation shocks or cocoon confinements, and also the conversion efficiency from hydrodynamic energy to radiation. Due to these simplifications, our results are still at the qualitative level, but we find that $E_{j(i s o)}$ is $\sim 10^{54} \mathrm{erg}$, while $L_{p(i s o)}$ is $\sim 10^{52} \mathrm{erg} / \mathrm{s}$, which are sufficiently large to explain GRBs. We would like to point out that, for the jet with for rapidly rotating progenitor (M150), large fraction of energy are radiated with high luminosity jet $\left(L_{j}>\right.$ $10^{50} \mathrm{erg} / \mathrm{s}$ ), while more than half of jet energy for M70 would be radiated with low luminous jet ( $L_{j}<5 \times 10^{49} \mathrm{erg} / \mathrm{s}$ ). According to these results, we conclude that neutrino-driven jet is capable to produce several types of outcomes such as GRBs, XRFs and also failed GRBs, by the difference of progenitor rotation.

\section{Summary}

We present numerical results of neutrino-driven jet propagation in a rotating Wolf-Rayet star. By changing the rate of progenitor rotation, we discuss the jet penetrability and their observational 


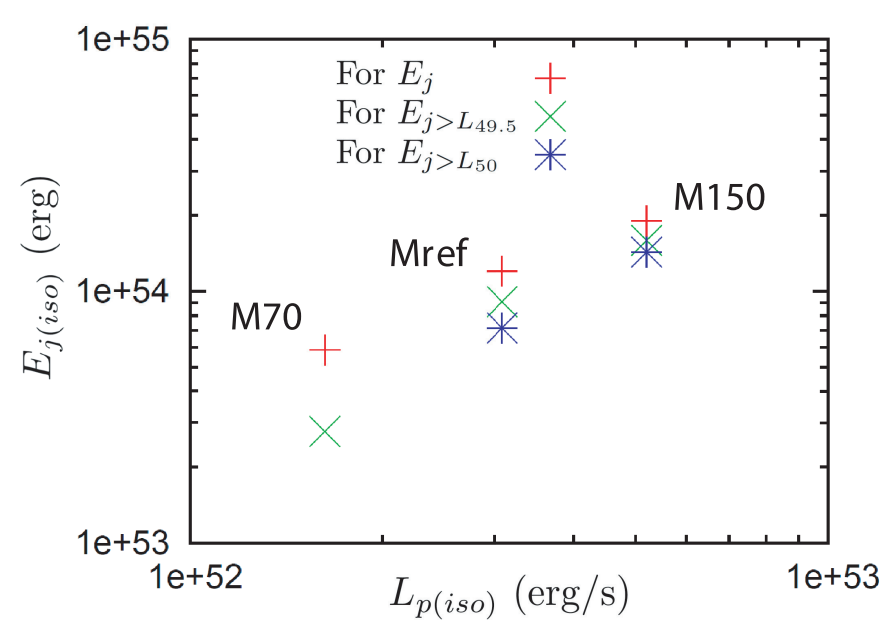

Figure 3: $E_{j(i s o)}$ and $L_{p(i s o)}$ relation among models with the successful jet breakout. $E_{j(i s o)}$ is calculated from $E_{j}$ (Red), $E_{j>L_{49.5}}$ (Green) and $E_{j>L_{50}}$ (Blue).

consequences by using analytic extrapolations for the stellar collapse. We show that every model except for M50 succeeds to break out the star. Especially, Mref or M150 has the jet with $L_{p(i s o)} \sim$ $10^{52} \mathrm{erg} / \mathrm{s}$ and $E_{j(i s o)} \sim 10^{54} \mathrm{erg}$, which are sufficiently large to explain GRBs. On the other hand, the energy in non-relativistic component $E_{d g}$ can be estimated around $\sim 10^{51} \mathrm{erg}$ which is roughly one order of magnitude lower than the typical explosion energy of Hypernova. Therefore, we may need extra mechanisms to explain the link between the GRBs-Hypernovae. We also find that model M50 can not successfully produce the jet breakout, so this model corresponds to the failed GRBs. It is interesting to note that, in spite of the successful jet break out for model M70, more than half of its total jet energy would be radiated with low luminosity jet $\left(L_{j}<5 \times 10^{49} \mathrm{erg} / \mathrm{s}\right)$. Therefore we expect that it could be observed as the low luminosity GRBs or XRFs.

\section{References}

[1] A. MacFadyen and S. E. Woosley, Collapsars - Gamma-Ray Bursts and Explosions in "Failed Supernovae, ApJ 524 (1999) 262

[2] R. Popham, S. E. Woosley and C. Fryer, Hyper-Accreting Black Holes and Gamma-Ray Bursts, ApJ 518 (1999) 356 [arXiv:astro-ph/9807028].

[3] I. Zalamea and A. M. Beloborodov, Neutrino heating near hyper-accreting black holes [arXiv:1003.0710]

[4] H. Nagakura, Y. Suwa and K. Ioka, Population III Gamma-Ray Bursts and Breakout Criteria for Accretion-Powered Jets, [arXiv:1104.5691].

[5] H. Nagakura, H. Ito, K. Kiuchi and S. Yamada, Jet propagations, breakouts and photospheric emissions in collapsing massive progenitors of long duration gamma ray bursts, ApJ 731 (2011) 80 [arXiv: 1009.2326$]$.

[6] S. Woosley and A. Heger, The Progenitor Stars of Gamma-Ray Bursts, ApJ 637 (2006) 914 [arXiv:astro-ph/0508175]. 
[7] D. Lopez-Camara, W. H. Lee and E. Ramirez-Ruiz, Astrophys. J. 716, 1308 (2010) [arXiv:0911.1336 [astro-ph.HE]].

[8] Y. Suwa and K. Ioka, Can Gamma-Ray Burst Jets Break Out the First Stars?, ApJ 726 (2011) 107 [arXiv:1009.6001]. 